\title{
GP5 Gene
}

National Cancer Institute

\section{Source}

National Cancer Institute. GP5 Gene. NCI Thesaurus. Code C126555.

This gene is involved in blood coagulation. 